\title{
VIDA UNIVERSITÁRIA E ATIVIDADE FÍSICA: UM ESTUDO SOBRE A PRODUÇÃO ACADÊMICA
}

\author{
UNIVERSITY LIFE AND PHYSICAL ACTIVITY: A STUDY ON THE ACADEMIC PRODUCTION
}

\section{Gustavo Marques Porto Cardoso ${ }^{a}$, Renata Meira Veras ${ }^{b}$, Maria Thereza Ávila Dantas Coelhoc ${ }^{c}$, Wilton Nascimento Figueredo ${ }^{d}$}

\author{
agugampc@hotmail.com, brenatameiraveras@gmail.com, 'therezacoelho@gmail.com, denfer.willfigueredo@gmail.com \\ Universidade Federal da Bahia - Salvador (BA), Brasil
}

Data de recebimento do artigo: 05/02/2017

Data de aceite do artigo: 27/04/2017

\section{RESUMO}

Introdução: A crescente visibilidade da atividade física na sociedade projeta a necessidade de discutila nas mais variadas populaçôes. Objetivo: Este estudo busca discutir a produçâo científica nacional e internacional sobre a atividade física em estudantes universitários. Materiais e Métodos: Como estratégia metodológica se optou por uma abordagem qualitativa, utilizando o estado da arte, para analisar os artigos sobre a temática contidos nas bases de dados SciELO e LILACS. Resultados: Fica evidenciado que a atividade física é abordada sob diversas óticas temáticas baseadas em estudos quantitativos na área da epidemiologia, majoritariamente centrados na região Sul do país, principalmente nas instituiçôes federais, com ápice de produção entre 2008-2012. Conclusão: Os autores e acadêmicos concordam sobre os benefícios da atividade física para a comunidade universitária. Porém, nota-se que os discentes ainda possuem baixo nível de prática de atividade física, havendo necessidade de implementação de mais programas e políticas de promoção de saúde para essa população, para que sejam futuros propagadores de um estilo de vida saudável.

Palavras-chave: Atividade física; universidade; saúde; promoção da saúde.

\section{ABSTRACT}

Introduction: Physical activity is gaining visibility in society and thus, it is necessary to discuss it in the most varied populations. Objective: This study aims to discuss the national and international scientific production on physical activity in university students. Materials and methods: As a methodological strategy, we opted for a qualitative approach, using the state of the art to analyze the articles contained in the SciELO and LILACS databases on the subject. Results: It is evidenced that physical activity is approached in several thematic perspectives, based on quantitative studies, in the epidemiology area, mostly focused on the southern region of Brazil and mainly in the federal institutions, having its apex between 2008 and 2012. Conclusion: Authors and scholars agree on the benefits of physical activity for the university community, but it is noted that the students still have a low level of physical activity, which shows a need to create more health promotion programs and policies for this population. These are the future propagators of a healthy lifestyle.

Keywords: Physical activity; universities; health; health promotion. 


\section{Introdução}

A discussão da atividade física relacionada à promoção da saúde é crescente nos últimos anos, como evidenciam os trabalhos científicos não só relacionados a universitários, mas a toda a população ${ }^{1-4}$. O progresso do interesse acerca do tema é atribuído às mudanças no comportamento social e individual da populaçáo, principalmente entre os jovens em idade universitária, cujo índice de sedentarismo cresce a cada dia ${ }^{5}$.

No Plano Plurianual do Ministério da Saúde se reconhece como meta programática a indução de atividade física/práticas corporais, mostrando a relevância do problema do sedentarismo ${ }^{1}$.

Nesse sentido, ressalta-se que a atividade física tem papel importante na promoção de saúde, devido às mudanças que sua prática pode proporcionar ao estilo de vida dos indivíduos. Consequentemente, a partir do início da década de 1990, a atividade física tem sido priorizada nos estudos de saúde pública, em razão de sua inserção nas agendas de saúde de diferentes países ${ }^{3}$.

No Brasil, apesar de diversos estudos atestarem os benefícios da atividade física para a saúde, estima-se que cerca de $60 \%$ da populaçáo é inativa ou insuficientemente ativa ${ }^{6}$. Nota-se que entre os estudantes universitários esses números não se alteram muito. Um estudo realizado com discentes da cidade de Fortaleza (CE) constatou sedentarismo em $70,3 \%$ da população da pesquisa ${ }^{7}$.

Considera-se, portanto, que a prática de atividade física está ligada intimamente ao estilo de vida escolhido pelo indivíduo e às suas condições de vida, pois esses hábitos dependem de uma série de fatores ambientais, sociais, demográficos e culturais que podem influenciar e/ou determinar o seu comportamento. De acordo com Azevedo et al. ${ }^{8}$, hábitos praticados na vida adulta, em sua maioria, são influenciados por outros adquiridos na adolescência, especialmente a prática de atividade física. Para grande parte dos estudantes universitários, o período de transição da adolescência para a idade adulta coincide justamente com a fase em que deixam o ensino médio para ingressar no meio acadêmico ${ }^{8}$.

Afirma-se que é no meio universitário, multirreferenciado, que o jovem está mais suscetível a influências para aquisição ou não de hábitos de vida saudáveis? Corroborando com essa ideia, Silva e Petroski ${ }^{10}$ afirmam que a universidade é a primeira experiência de liberdade para a maioria dessa população, na qual surgem questionamentos sobre valores, crenças e atitudes que foram empregadas pela família durante sua educação, podendo promover consequentes mudanças no estilo de vida.
Estudos afirmam que a prática de atividade física regular e a mudança de velhos hábitos atuam na prevenção de diversas doenças crônicas não transmissíveis (DCNT) e melhoram a qualidade de vida dos praticantes ${ }^{11,12}$.

A temática acerca da atividade física em universitários se apresenta multifatorial e complexa, enfatizando-se, portanto, a necessidade de uma análise da bibliografia científica sobre a importância da atividade física na promoção de saúde dessa população.

Portanto, este estudo objetiva mapear e discutir a produçâao científica a partir de artigos já publicados acerca da atividade física em universitários, no cenário científico nacional e internacional.

\section{Materiais e métodos}

Este é um trabalho de caráter bibliográfico denominado "estado da arte", qualitativo. O "estado da arte" tem o objetivo de mapear e discutir determinado tema nas suas diversas dimensões do conhecimento, em que por meio de categorias e subcategorias é possível analisar, em cada trabalho e no conjunto, as abordagens dos fenômenos estudados, obtendo ou não respostas sobre os estudos ${ }^{13}$. Para Romanowski e Ens ${ }^{14}$, o estado da arte é uma importante ferramenta para o campo teórico de uma área do conhecimento, pois colabora para a identificação das suas restriçôes, lacunas e conceitos, apontando direção a novas pesquisas.

Para a realização deste estudo, foram utilizadas duas bases de dados eletrônicas, sendo elas a Scientific Electronic Library Online (SciELO) e a Literatura Latino-Americana e do Caribe em Ciências da Saúde (LILACS). A coleta foi realizada no dia 22 de novembro de $2013 \mathrm{em}$ ambas as bases, nas quais foram utilizados os descritores "atividade física" e "universitários".

Metodologicamente, a leitura dos artigos se deu em três momentos: a primeira foi realizada a partir dos resumos, com os quais se observou a concordância com o tema proposto; no segundo momento, os artigos selecionados foram lidos por completo para a construção de tabelas; por último foi realizada uma nova leitura para a interpretação das ideias dos autores.

Para a exclusão de artigos que não seriam utilizados neste estudo foram definidos cinco critérios: a) não estar em formato de artigo, a exemplos de teses, editoriais, dissertaçóes etc.; b) a repetição dos artigos na mesma base de dados ou em outra; c) o não acordo dos artigos com a temática proposta; d) o não pertencimento a revistas de open access; e) erro de página na versão on-line. 


\section{Resultados e discussão}

Como resultado, foram obtidos 118 estudos no total, sendo 43 na base de dados da SciELO e 75 na base do LILACS. Dos artigos já selecionados, foram encontrados três idiomas: no SciELO dois em espanhol, três em inglês e 15 em português; e no LILACS cinco em espanhol, três em inglês e 18 em português. Portanto, à luz dos critérios de inclusão e exclusão, este estudo será composto por 46 artigos (Tabela 1).

Tabela 1: Resultado da distribuição dos artigos.

\begin{tabular}{lccc} 
& SciELO & LILACS & Total \\
\hline Utilizados & 20 & 26 & 46 \\
Excluídos & 23 & 49 & 72 \\
TOTAL & 43 & 75 & 118 \\
\hline
\end{tabular}

Fonte: Elaboração própria a partir dos dados coletados.

A partir das leituras e análises dos textos selecionados para a elaboração deste estudo, foram criadas categorias e subcategorias, no intuito de discutir os artigos quanto à temática, à metodologia, ao campo disciplinar, à inserçáo dos autores e ao ano de publicaçáo. A Tabela 2 apresenta as subcategorias que foram concebidas por meio da análise dos temas dominantes em cada artigo e serão discutidas separadamente.

Tabela 2: Categorias e subcategorias dos artigos selecionados.

\begin{tabular}{ll}
\multicolumn{1}{c}{ Categorias } & \multicolumn{1}{c}{ Subcategorias } \\
1. Temático-conceitual & $\begin{array}{l}\text { a) Atividade física em universitários } \\
\text { b) Estado nutricional } \\
\text { c) Imagem corporal } \\
\text { d) Estilo de vida } \\
\text { e) Prevenção de doenças }\end{array}$ \\
& $\begin{array}{l}\text { a) Abordagem qualitativa } \\
\text { b) Abordagem quantitativa } \\
\text { c) Abordagem quali-quantitativa }\end{array}$ \\
\hline 3. Teórico-metodológico & \\
4. Inserçâao institucional dos autores & \\
\hline 5. Ano de publicaçáo & \\
\hline
\end{tabular}

Fonte: Elaboração própria a partir dos dados coletados.

\section{Temático-conceitual}

Esta categoria surgiu da necessidade de analisar as principais temáticas e linhas de pesquisas trabalhadas nos artigos selecionados para esta revisão. Assim, para análise dos dados dessa categoria, foram elencadas cinco subcategorias: atividade física em universitários, estado nutricional, imagem corporal, estilo de vida e prevenção de doenças (Gráfico 1), que serão discutidas a seguir.
Gráfico 1: Análise dos artigos por eixo temático conceitual

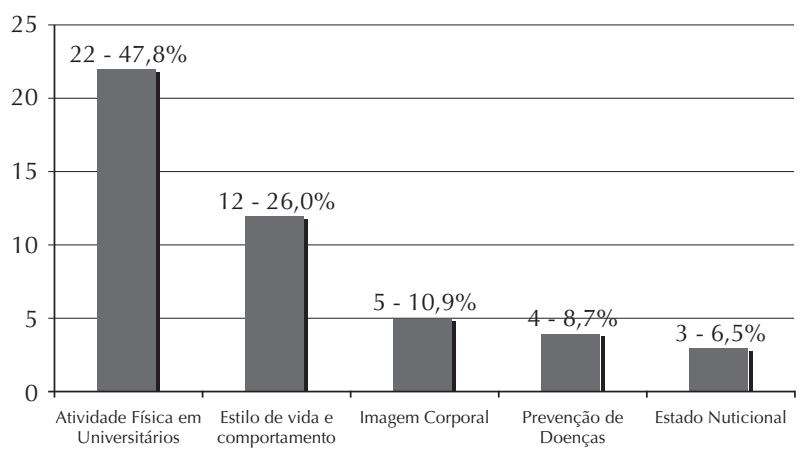

Fonte: Elaboração própria a partir dos dados coletados.

\section{Atividade física em universitários}

Atividade física é uma expressão ampla que pode ser definida como qualquer movimento corporal, produzido pelos músculos esqueléticos, que resulta em gasto energético maior do que os níveis de repouso. Já o exercício físico (um dos seus principais componentes), é uma atividade física planejada, estruturada e repetitiva que tem como objetivo final ou intermediário aumentar ou manter a saúde/aptidão física ${ }^{15}$.

Assim, a partir da análise, verificou-se que, dos textos nas duas bases de dados, 22 centralizaram suas discussôes acerca da atividade física dos universitários.

Observa-se que no biênio 2006-2007 houve a inclusão da atividade física como uma das principais estratégias das agendas de saúde pública nacional e internacional, o que pode justificar o grande número de artigos publicados com essa temática ${ }^{16}$. Segundo Malta et al. ${ }^{1}$, a Política de Promoção de Saúde incentiva as práticas corporais, pois reconhece a relevância epidemiológica do sedentarismo, promovendo o compromisso social e institucional de adoçáo de modos de vida mais saudáveis.

Verificou-se na análise dessa subcategoria que os trabalhos tendem a oferecer uma discussão voltada para a epidemiologia, levando a discussão da atividade física como fator preventor das DCNT.

Os universitários pesquisados em uma instituição da cidade de Curitiba, quando comparados por sexo, apresentam níveis de atividade física diferentes, sendo que o sexo masculino apresenta um nível maior de atividade física que os do sexo feminino ${ }^{12}$. Esses resultados também foram encontrados em outro estu$\mathrm{do}^{11}$ que, por meio de análise com estudantes da área de saúde da Universidade de Brasília, comprovou um nível de práticas suficientes entre os alunos, em que o sexo masculino apresentou níveis maiores que o feminino, assim como também demonstraram outros estudos ${ }^{10,17,18}$. 
Quando se relaciona a temática do texto ao nível de inatividade física, Quadros et al. ${ }^{19}$ sugerem que existe uma tendência maior à prática de atividades físicas no sexo masculino, tendo as mulheres percentuais maiores de sedentarismo.

Ainda sobre o sedentarismo, Marcondelli, Costa e Schmitz ${ }^{11}$ relacionam a falta de tempo, os trabalhos acadêmicos e a falta de dinheiro como principais motivos que levam à inatividade física por parte dos estudantes. Ressalta-se que a maioria dos estudantes entrevistados não possuía renda fixa e ainda era sustentada por familiares.

Por outro lado, grande parte dos artigos integrantes dessa subcategoria analisa a atividade física como fator indispensável na prevenção das DCNT. Guedes, Legnani e Legnani ${ }^{20}$, ao estudarem os motivos para a prática de atividade física nos universitários da Universidade Estadual de Londrina, chegam à conclusão que o principal motivo para a prática é a prevençáo de doenças, seguida da melhora da condição física e do controle do peso corporal. Esse estudo indica que a visão da prática de atividade física, tanto entre os pesquisadores como nos universitários, ainda está relacionada ao modelo biomédico do processo saúde-doença, "caracterizado pela ênfase nos aspectos biológicos, individuais e pela abordagem mecanicista [...] passa[ndo] a fragmentar o corpo em sistemas, órgãos, tecidos e células, estruturando um conhecimento cada vez mais especializado sobre cada função orgânica" ${ }^{21}$. Trata-se de modelo, portanto, no qual a atividade física tem função preventiva ou curativa nos indivíduos.

Outros temas são abordados por autores, como o bem-estar psicológico e a adiposidade corporal ${ }^{22}$; a prática desportiva e suas relaçôes com as autopercepçóes físicas, bem-estar subjetivo e felicidade ${ }^{23}$; a relação entre atividade física e lazer ${ }^{24}$; e a análise da prática esportiva com pessoas portadoras de deficiência visual ${ }^{25}$.

A avaliação dos artigos nessa subcategoria indica que os pesquisadores ofereceram diversos olhares para a prática de atividade física em universitários, permitindo um mapeamento dos níveis de atividades da população universitária. Verifica-se também que os autores realizaram suas pesquisas sob o enfoque da epidemiologia. Segundo Hallal e Knuth ${ }^{15}$, essa tendência se dá pelo fato de a produção científica relacionada à atividade física estar associada aos centros de pesquisa em epidemiologia e saúde coletiva, onde os estudos acabam tendo um viés mais quantitativo. Esses autores ainda relatam a necessidade da aproximação dessa temática com a abordagem qualitativa, principalmente se estiverem relacionadas com o campo da educação física e das suas subáreas, pois o modelo epidemiológico por si só não é capaz de responder aos questionamentos do campo.

\section{Estilo de vida}

Nesta subcategoria serão elencados os artigos que apresentaram como temática principal a atividade física sob a ótica da mudança do estilo de vida dos universitários à luz do pentáculo do bem-estar ${ }^{26}$, que possui como componentes integradores deste modelo a nutrição, o estresse, os relacionamentos, a atividade física e o comportamento preventivo. Do universo de 46 artigos deste estudo, 12 fazem parte desta subcategoria.

A atividade física apresenta-se como um fator preponderante na mudança comportamental da população mundial, pois, a partir da escolha de um estilo de vida mais ativo, outros fatores da mudança de comportamento surgem, como a melhora dos hábitos alimentares, a diminuição do tabagismo e do consumo etílico, a diminuição do estresse, entre outros? .

Colares, Franca e Gonzalez ${ }^{27}$, ao estudarem a diferença entre os sexos e as condutas de saúde com alunos dos cursos de saúde da Universidade Estadual de Pernambuco, concluíram que, apesar dos homens apresentarem maior nível de prática de atividades físicas, as mulheres se preocupam mais com a alimentação e outros fatores relacionados à promoçáo da saúde. Assim, foi demonstrado que os homens e mulheres possuem níveis de atividade física diferentes, sendo que as mulheres apresentam frequências de atividades menores e níveis de condutas também diferentes, corroborando os dados discutidos por outros autores, como Silva et al. ${ }^{18}$, Marcondelli, Costa e Schmitz ${ }^{11}$, Rodrigues, Sheik e Mayer ${ }^{17}$, Silva e Petroski ${ }^{10}$, Cieslak et al. ${ }^{12}$, entre outros. Para esses autores, o fato de os indivíduos de sexos diferentes apresentarem condutas de saúde divergentes faz que as estratégias de promoção também sejam diferenciadas.

Por outro lado, os resultados de uma pesquisa com estudantes da área de saúde no início e no final do curso, que relacionava o tempo de permanência na universidade e a aquisição de condutas saudáveis pelos estudantes, demonstraram que não há diferenças significativas entre esses fatores em ambos os sexos. Corroborando esses dados, Brandáo, Pimentel e Cardoso ${ }^{28}$, ao avaliarem o impacto da exposição acadêmica para a saúde, concluíram que o nível de instrução não muda as condutas de saúde dos estudantes, mesmo naqueles que são da área de saúde, tanto do sexo feminino como do sexo masculino. Esse dado se destaca uma vez que estima-se que futuros profissionais da área deveriam mudar suas atitudes em relação aos hábitos de saúde devido à aquisição de conhecimentos ao longo do curso ${ }^{29}$.

Quanto aos fatores que mais determinam a saúde no meio universitário - em um estudo bibliográfico realizado por fisioterapeutas da Universidade Del Rosário, Colômbia, sobre os determinantes do estilo de vida e sua 
implicação na saúde dos estudantes - foram apontados a atividade física, os hábitos alimentares e o consumo de tabaco e álcool como fatores mais determinantes no estado de saúde dos indivíduos. Os autores concluem que a boa intervençáo nesses quatro fatores permitem mudanças drásticas no comportamento dos universitários, propondo como uma das açôes a inclusão da atividade física como componente curricular nas universidades, a melhoria da oferta e do acesso a uma alimentaçáo saudável e campanhas que combatam o consumo de tabaco e álcool entre essa população ${ }^{30}$.

O que se apresenta em comum nos trabalhos desta categoria é a concordância de que, em geral, os estudantes universitários não possuem um estilo de vida saudável, o que os coloca em situaçóes de riscos para o desenvolvimento de vários agravos à saúde. Portanto, são necessárias ações promotoras da saúde no meio acadêmico para a mudança dessas condutas.

\section{Imagem corporal}

Nos últimos anos, os meios de comunicação em massa, principalmente a televisão, vêm mostrando corpos esculturais como sinônimo de saúde e beleza, influenciando jovens e adultos de ambos os sexos a buscarem o padrão corporal determinado socialmente.

Antes de discutir os achados dos autores nesta subcategoria, faz-se necessário conceituar o que se entende por imagem corporal: "a formação multidimensional que envolve a percepção corporal, o desenho que a pessoa tem em sua mente em relação ao tamanho, imagem e formas corporais, juntamente com os sentimentos que ela possui em relação a isso" ${ }^{\text {31 }}$.

Dos artigos analisados neste estudo, cinco possuíam como temática central a percepção dos universitários sobre a sua imagem corporal. A partir das conclusôes dos autores, evidencia-se a prevalência de insatisfação corporal por parte dos universitários.

$\mathrm{Na}$ pesquisa de Rech, Araújo e $\mathrm{Vanat}^{32}$ foi demonstrado o alto índice de insatisfação com a imagem corporal por parte dos estudantes. Esse fato se deve, principalmente, pelo aumento da ingestáo de alimentos hipercalóricos e a diminuição dos níveis de atividades físicas nessa população, gerando então uma balança de gordura positiva. Os autores afirmam que isso não é um problema apenas dos estudantes, mas dos indivíduos adultos de forma geral na sociedade moderna, sendo que em universitários que cursam educação física na Universidade Estadual de Ponta Grossa a insatisfação corporal foi de $61,2 \%$.

Por meio dos resultados apresentados nos artigos, conclui-se que a satisfação com a imagem corporal está atrelada a diversos fatores. Os resultados encontrados em estudos mais recentes mostram que os estágios de mudanças comportamentais estão entrelaçados com a satisfação ou insatisfação corporal e em relação à prática de atividade física, sabendo-se que homens e mulheres ativos possuem um grau de satisfaçáo maior com sua imagem $^{33}$.

Porém, em outro estudo, afirma-se que altos índices de insatisfação corporal podem ser apresentados em estudantes com moderados e altos níveis de atividade física, sendo que os fatores que mais contribuem para esta realidade são o sexo e o estado nutricional dos universitários ${ }^{32}$.

Ao relacionar o índice de massa corporal (IMC) e insatisfação com a imagem corporal, conclui-se que as variáveis sociodemográficas não têm relação com a autopercepção corporal. A maior insatisfação entre os homens está relacionada com a magreza, e nas mulheres, o sobrepeso, confirmando assim o desejo do corpo padrão imposto pelos meios de comunicação em massa ${ }^{5}$.

Evidencia-se, na maioria dos artigos, que a imagem corporal não está relacionada diretamente com os níveis de atividades físicas em universitários, enquanto a adoção de hábitos alimentares inadequados, a diferença entre os sexos, a internalizarão do ideal de beleza corporal, as influências do meio social e familiar e o IMC são fatores mais dominantes na insatisfação com a imagem corporal do que o nível de atividade física ${ }^{33}$.

\section{Prevenção de doenças}

A maioria dos autores dos estudos aqui analisados indica a prática de atividade física como condição fundamental para a prevenção das DCNT, porém, apenas quatro deles relacionaram sua pesquisa com doenças específicas.

Simão et al ${ }^{34}$ realizaram uma pesquisa na cidade de Lubango, Angola, e constataram, sob a ótica dos cinco elementos do campo da saúde (biologia humana, meio ambiente, estilo de vida, participação social e organização dos serviços de saúde), que os estudantes possuíam hábitos saudáveis em relação à prática de atividade física e que essa prática diminuía os fatores de risco para a aquisição de hipertensão arterial.

Em outro estudo, Vilarinho et al. ${ }^{35}$ analisaram os fatores de risco modificáveis para a ocorrência da diabetes mellitus tipo 2 (DMT2) em estudantes do $4^{\circ}$ período de enfermagem de uma instituição pública da cidade do Rio de Janeiro. Esses autores constataram a necessidade de criação, no universo acadêmico, de programas educacionais de promoção de saúde que orientem os alunos quanto aos riscos do sedentarismo e dos hábitos alimentares incorretos, agindo, assim, no combate às doenças como a DMT2. 
Por meio de análises clínicas, em um estudo com dez universitários adultos e ativos, foi constatado que em exercício físico máximo na situação pós-prandial (após a refeição) há alteraçôes dos níveis de triglicerídeos. No entanto, não ocorre a mesma diminuição nos valores da glicemia, proteínas, colesteróis totais e hematócrito. Portanto, esses autores não chegaram à conclusão de que esses exercícios diminuam a probabilidade do aparecimento de doença arterial coronariana ${ }^{36}$.

Quando foram analisados os biomarcadores séricos - entidades que podem ser medidas experimentalmente e indicam a ocorrência de uma determinada funçáo normal ou patológica de um organismo encontrada no sangue - ao nível de atividade física em universitários da Venezuela, Cordero et al. ${ }^{37}$ concluem que apenas uma pequena parte da amostra, composta por indivíduos sedentários, tinha baixa quantidade de albumina sérica, revelando que poucos indivíduos possuíam suscetibilidade a desenvolver doenças cardiovasculares.

Diante da discussão dos quatro estudos que compóem esta subcategoria, nota-se que nem todos os autores concordam que a prática da atividade física, por si só, contribui para a diminuição do aparecimento de doenças específicas. Assim, a saúde pode ser considerada como um processo multifatorial em que há a necessidade da soma desses fatores para que eles possam agir positivamente na melhora da qualidade de vida. Os autores concordam que há uma grande necessidade de se pesquisar na populaçáo dos estudantes universitários a atividade física como inibidora das DCNT, e que cabe às instituiçóes de ensino superior a criação de programas que promovam hábitos sadios no meio acadêmico.

\section{Estado nutricional}

Neste subitem foram agrupados três artigos nos quais os pesquisadores relacionam a atividade física com as condutas alimentares dos estudantes.

A entrada na vida universitária provoca alteraçóes nos hábitos alimentares dos acadêmicos devido à influência das novas redes sociais adquiridas e da adaptação à nova rotina. Ressalta-se também que essas mudanças estáo intimamente ligadas ao nível de atividade física ${ }^{11,29,38}$.

No estudo de Simáo et al. ${ }^{39}$ se observa que a alimentação dos estudantes universitários está pautada basicamente em alimentos ricos em açúcares e gorduras, devido à falta de tempo para fazer suas refeiçóes. Porém, em outro estudo, analisando o consumo de frutas e hortaliças por estudantes universitários do Rio Branco (AC), os pesquisadores concluem que a alimentação mais saudável está intimamente ligada à prática de atividade física, sendo que estudantes mais ativos tendem a consumir mais alimentos saudáveis. Concluíram também que com a prática de atividades há uma diminuição da ingestão de alimentos conhecidos como fast food $^{\beta 8}$.

Corroborando os estudos citados, destaca-se uma pesquisa sobre o consumo excessivo de produtos de origem animal, como o leite e a carne, pelos estudantes. Os resultados indicam que os indivíduos com um estilo de vida mais ativo se preocupam em consumir mais alimentos dessa natureza, devido ao aporte de proteínas contidas nesse grupo alimentar ${ }^{40}$.

Quando foram analisados os dados da comparação dos alunos das demais áreas com os estudantes da área de saúde (que os autores consideram disseminadores de um estilo de vida saudável) esse panorama não se altera, pois estes praticam pouca atividade física e têm uma alimentação pouco balanceada ${ }^{41}$.

Assim, como afirmam Madureira et al. ${ }^{42}$, a universidade tem papel fundamental na mudança no estilo de vida dos estudantes, com a obrigaçáo de promover açôes para a melhora da qualidade de vida e também da alimentação.

Diante dos dados evidenciados, é necessária a adoção de estratégias eficazes de promoção de saúde para alteração do panorama alimentar em que se encontram os estudantes: alimentaçáo empobrecida de nutrientes e rica em açúcares e gorduras. Ressalta-se, portanto, a importância da influência social para as mudanças significativas desse padrão alimentar.

\section{Teórico-metodológico}

Para Reis, Ciconelli e Faloppa ${ }^{43}$, a pesquisa científica se pauta em um agrupamento de procedimentos, que de forma sistemática e dentro de um raciocínio lógico, tem como principal objetivo encontrar uma estratégia que solucione, por meio de métodos científicos, os problemas propostos.

Dessa forma, os autores demonstram a importância da escolha da abordagem metodológica como fator primordial para a construção de um trabalho científico, sendo esta indiscutível para a obtenção de um rigor científico nos estudos sobre os mais variados assuntos. Portanto, nesta categoria se discute os artigos quanto à sua abordagem metodológica.

A abordagem metodológica quantitativa foi a escolha dos autores de 44 artigos, enquanto dois optaram por abordagens diferentes: um pela abordagem qualitativa e um pela abordagem quali-quantitativa.

Segundo Turato $^{44}$ a revisão narrativa de literatura é um instrumento comum na abordagem qualitativa, onde os autores discutem vários trabalhos que se relacionam a um tema previamente escolhido à luz de um rigor científico e metodológico, analisando o material 
selecionado. Nessa perspectiva se enquadra o trabalho de García-Laguna et al. ${ }^{30}$, destacando, por meio da revisão de literatura, os mais relevantes hábitos dos estilos de vida dos estudantes universitários, que seriam a atividade física, os hábitos alimentares, o consumo de tabaco e o consumo nocivo de álcool. Os mesmos autores identificaram também que fatores influenciaram a aquisição ou não desses hábitos na população estudada e as estratégias utilizadas para as mudanças de comportamentos geradores de fatores de risco para a saúde. Ainda segundo Turato ${ }^{44}$, trabalhos relacionados a fatores, barreiras e pontes facilitadoras no processo saúde-doença estão intimamente ligados com o método qualitativo.

A abordagem quali-quantitativa, para Landim et al. ${ }^{45}$, é a junção dos dois caminhos metodológicos e, ao escolher estudar sobre a ótica das duas abordagens, o pesquisador "é, pois, conduzido no sentido de integrar as vantagens do método quantitativo com as do qualitativo". Essa escolha metodológica está apresentada no estudo de González e Tamayo ${ }^{46}$, em que caracterizam os obstáculos cognitivo-emotivos que estão relacionados à prática da atividade física regular e qual o significado desses obstáculos para os universitários; utilizando instrumentos quantitativos, como o International Physical Activity Questionnaire (IPAQ), e qualitativos, como o instrumento do grupo focal, unindo os resultados para a construção da conclusão.

Percebe-se que a maioria dos autores analisados optou pela abordagem quantitativa como metodologia. Para Turato ${ }^{44}$, as pesquisas que valorizam a frequência, a incidência e a prevalência em um intervalo de tempo em uma população são caracterizadas como de abordagem quantitativa. Portanto, os autores que optaram por essa abordagem analisam numericamente os valores anteriormente relacionados.

Assim, verifica-se que o olhar epidemiológico da maioria dos autores estudados neste estado da arte pode justificar a predominância da abordagem quantitativa para essa temática.

\section{Campo disciplinar}

Nesta categoria, a discussão será acerca do campo disciplinar a que os artigos analisados neste estudo pertencem.

Segundo Bourdieu ${ }^{47}$, o campo científico é um espaço de jogo que se apresenta de forma concorrente, derivada de um sistema de relaçóes a partir de posições adquiridas por meio de conflitos anteriores. Nesse campo, os pesquisadores disputam o monopólio de competência científica, em que as regras são ditadas por seus participantes ou pares ${ }^{48}$.
A partir da ideia de campo científico de Bordieu, Almeida Filho ${ }^{49}$ define campo disciplinar como um "espaço histórico-social e institucional", no qual a concepçáo de disciplina decorre da organização e da delimitação, selecionando conhecimentos importantes para a área em questáo.

Dos artigos analisados, a grande maioria norteia-se a partir do campo disciplinar da epidemiologia.

Desde a antiguidade, terapeutas gregos e chineses já ressaltavam a importância da atividade física para a melhoria da saúde e o tratamento de doenças. Já se imaginava a existência de uma relação estreita entre as práticas corporais e a melhoria da saúde; porém essa relação só se tornou evidente com a era epidemiológica das DCNT, quando o sedentarismo apareceu como fator importante dos agravos da saúde. É nesse momento, com a transição epidemiológica, que há a inversão das causas de morte das doenças infecciosas para as doenças cardiovasculares $^{50}$.

Levando em consideração a relação entre práticas corporais e saúde, os estudos epidemiológicos em atividade física, segundo Pitanga ${ }^{50}$, podem ser classificados em: observacionais e intervenção, em que estudos observacionais subdividem-se em transversais, caso-controle (retrospectivos), coorte (prospectivos) e ecológicos; e os estudos de intervenção, experimentais (ensaios clínicos).

Segundo a classificaçãa anteriormente citada, diversos autores abordam esses métodos na construção de seus estudos, portanto acredita-se que suas pesquisas compóem o campo epidemiológico.

Entre os estudos classificados como observacionais, temos o de Cieslak et al. ${ }^{12}$, que possui caráter transversal por analisar a qualidade de vida e o nível de atividade física entre estudantes de uma universidade pública brasileira; o estudo de Madureira et al. ${ }^{42}$ no qual utilizam do mesmo método para verificar a associação de mudança comportamental e o estado nutricional em universitários da Universidade Federal de Santa Catarina; o estudo de Rech, Araújo e Vanat ${ }^{32}$, no qual analisam a autopercepção da imagem corporal em acadêmicos de educação física da Universidade Estadual de Ponta Grossa, entre outros. Reitera-se que a maioria dos autores analisados utiliza o método transversal como procedimento metodológico para a obtenção de dados.

Outros métodos observacionais também são utilizados nos artigos aqui discutidos. Como exemplos citamos a amostra estratificada no trabalho de Franca e Colares $^{29}$, o estudo longitudinal no artigo de Brandão, Pimentel e Cardoso ${ }^{28}$, o estudo seccional no caso de Fontes e Vianna ${ }^{51}$, o estudo de caso na pesquisa de Rigoni et al. ${ }^{6}$

Nos estudos classificados como de intervenção, pode-se tomar como exemplo a pesquisa de Belli et al. ${ }^{36}$, em que os autores analisam o comportamento dos 
triglicérides e verificam glicemia, colesterol e proteínas totais em situação pós-prandial na presença de exercícios físicos agudos em universitários adultos.

Portanto, os artigos estudados assentam-se no campo disciplinar da epidemiologia, o que se justifica por meio das escolhas e estratégias metodológicas, pelo tipo de obtençấo dos dados e pelos objetivos traçados dos autores.

\section{Inserção institucional dos autores}

A discussão da inserção institucional dos autores dos artigos selecionados, por meio das categorias administrativas e locais, faz-se necessária para compreender como se apresenta a produção científica sobre o tema pré-definido segundo a sua localização.

Gráfico 2: Análise da inserção dos autores por nacionalidade.

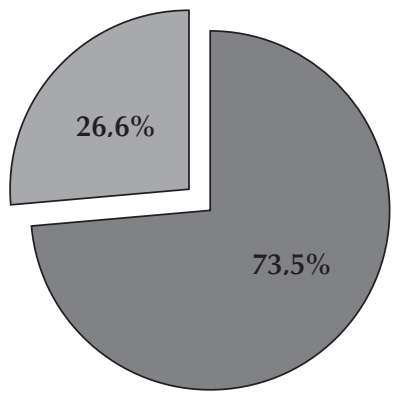

Fonte: Elaboração própria a partir dos dados coletados.

O Gráfico 2 demonstra que a produção brasileira, vista a partir da inserçấo institucional dos autores dos artigos aqui analisados, corresponde a $73,5 \%$, enquanto a produção internacional equivale a $26,6 \%$. Observa-se, então, que a atividade física nos estudantes universitários vem sendo discutida de forma ampla no país, assim como, no cenário internacional.

Figura 1: Análise da inserção dos autores por região brasileira.

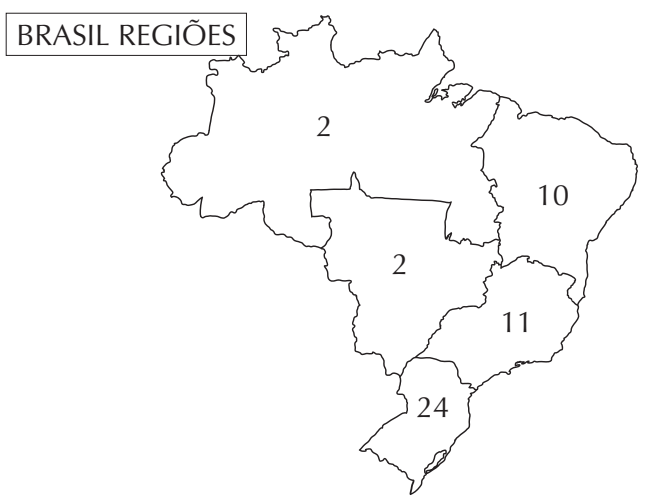

Fonte: Elaboração própria a partir dos dados coletados.
Observando a produçáo brasileira à luz da regionalização (Figura 1), pode-se inferir que a produção científica está centralizada nas regiôes Sul e Sudeste do país, somando 35 autores, o que mostra sua hegemonia na pesquisa científica do Brasil. Dois fatores podem contribuir para esse cenário: o maior número de instituiçóes de ensino superior - consequentemente maior quantidade de cursos e grupos de pesquisa - e a maior disponibilidade de recursos humanos/incentivos à pesquisa.

Gráfico 3: Análise da inserção dos autores por categorias administrativas.

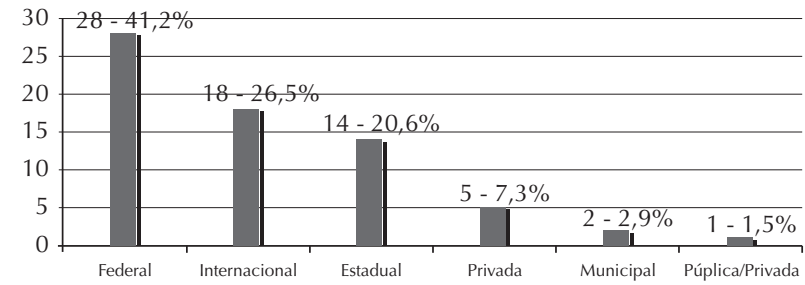

Fonte: Elaboraçăo própria a partir dos dados coletados.

Conforme se observa no Gráfico 3, a maior produção sobre a temática está concentrada na categoria administrativa de âmbito federal. Acredita-se que isso ocorra devido ao maior incentivo à pesquisa dada pelo Governo Federal para essas instituições.

\section{Ano de publicação}

Considerar o ano de publicação se faz necessário, uma vez que se evidencia a primazia por determinado tema em um dado ano. No Gráfico 4 nota-se a distribuição dos artigos por ano de publicação.

Gráfico 4: Quantidade de artigos por ano de publicação.

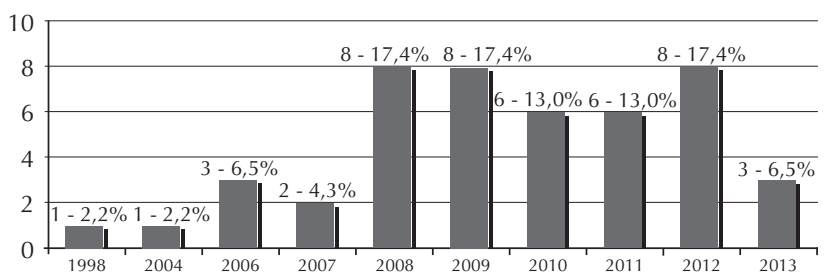

Fonte: Elaboração própria a partir dos dados coletados.

A análise desses dados em um contexto mundial levanta a hipótese de que o crescente número de trabalhos científicos produzidos sobre a temática, a partir de 2008, é devido à intensificação da transição epidemiológica que vem ocorrendo no planeta. Neste estudo, entende-se transição epidemiológica a partir do conceito de Omram ${ }^{52}$, que relaciona essas mudanças com as alterações ocorridas nos padrões de morte, mortalidade e invalidez em uma população. Esse processo possui a 
característica da mudança do perfil epidemiológico das doenças transmissíveis para as não transmissíveis, e vem ocorrendo de forma mais significativa nos últimos anos.

É notório que os pesquisadores da área de saúde estão direcionando seus estudos para os fatores modificáveis dessa transiçáo, sendo um desses o combate à inatividade física, salientando a necessidade da prática regular de atividade física.

Após 85 anos de formação da primeira turma de professores de educaçáo física, existem hoje mais de 800 cursos no país, e, com isso, houve um consequente aumento na quantidade de grupos de pesquisa na área ${ }^{53}$. Esse quadro se ampliou desde a criação de alguns programas pelo Governo Federal para aumentar o número de vagas nas instituiçóes de ensino superior e financiar os estudos de populações em estado socialmente vulneráveis, tais como o Programa de Financiamento do Estudante (Fies) e o Programa de Apoio a Planos de Reestruturação e Expansão das Universidades Federais (Reuni). Esses programas tornaram mais democrático o acesso à educação superior, tanto na rede particular como na rede pública de ensino.

Portanto, observa-se que a transição epidemiológi$\mathrm{ca}$, que ocorre a passos largos no Brasil, influencia no aumento do interesse dos pesquisadores sobre os fatores modificáveis para a prevenção de DCNT. Por outro lado, o aumento de vagas nos cursos superiores brasileiros, o consequente aumento de vagas nos cursos de educação física e a ampliação do número de grupos de pesquisas nas temáticas referentes à área provavelmente justificam o maior número de publicações após 2008.

\section{Conclusão}

Este estudo forneceu o cenário das publicaçóes sobre a atividade física em universitários. Notou-se que os autores trabalhados neste artigo relacionaram a atividade física em estudantes universitários com diferentes elementos que podem ou não influenciar nesta prática. Fatores como tabagismo, álcool, estado nutricional, condição socioeconômica e nível de instrução, entre outros, foram utilizados, esboçando-se, então, um panorama da vida universitária e a sua relação com a atividade física.

Constata-se que quase a totalidade dos autores aqui pesquisados desenvolveram seus estudos à luz da epidemiologia. Contudo, esse campo disciplinar não fornece respostas a todas as perguntas que o tema exige, sendo necessária a realização de outros trabalhos que discutam a temática sob a ótica de outros campos da ciência.

Entende-se que diversas instituiçōes de ensino superior, tanto nacionais quanto internacionais, estáo produzindo trabalhos sobre a atividade física em universitários. Isso provavelmente ocorre devido ao crescente número de instituiçóes criadas e o consequente aumento quantitativo de alunos no ensino superior, bem como o agravamento da transição epidemiológica na sociedade moderna - fatores esses que geram maior interesse sobre o assunto.

Portanto, este trabalho evidencia que os autores estudados concordam entre si sobre os benefícios da atividade física para a comunidade universitária, como a mudança do estilo de vida e a consequente melhoria da saúde, e que o sedentarismo pode causar sérias complicaçôes no processo saúde-doença, surgindo a necessidade de políticas que promovam a mudança comportamental desses estudantes, futuros profissionais de saúde. No entanto, é necessário salientar que, por utilizar duas bases de dados (SciELO e LILACS), os resultados não representam por completo toda a produçáo científica sobre a temática em questão, sendo exigida uma pesquisa mais aprofundada em outras bases de dados para a análise de mais referências sobre o tema.

\section{Referências}

1. Malta DC, Castro AM, Gosch CS, Cruz DKA, Bressan A, Nogueira JD, et al. A Política Nacional de Promoção de Saúde e a agenda da atividade física no contexto do SUS. Epidemiol Serv Saúde. 2009;18(1):79-86.

2. Ferreira MS, Najar AL. Programas e campanhas de promoção da atividade física. Ciênc. Saúde Coletiva. 2005;10Suppl:207-19.

3. Nahas MV. Atividade física, saúde e qualidade de vida. Londrina: Midiograf; 2006.

4. Mendonça BCA, Toscano JJO, Oliveira ACC. Programa academia da cidade de Aracajú: promovendo saúde por meio da atividade física. Rev Bras Ativ Fís Saúde. 2009;14(3):211-6.

5. Ferrari EP, Petroski EL, Silva DAS. Prevalence of body image dissatisfaction and associated factors among physical education students. Trends Psychiatry Psychother. 2013;35(2):119-27.

6. Rigoni, PAG, Junior JRAN, Costa GNFS, Vieira LF. Estágios de mudança de comportamento e percepção de barreiras para a prática de atividade física em universitários do curso de Educação Física. Rev Bras Ativ Fís Saúde. 2012;17(2):87-92.

7. Veras VS, Monteiro LZ, Landim CAP, Xavier ATF, Pinheiro MHNP, Junior RMM. Levantamento dos fatores de risco para doenças crônicas em universitários. Rev Bras Prom Saúde. 2007;20(3):168-72.

8. Azevedo MR, Araújo CL, Silva MC, Hallal PC. Tracking of physical activity from adolescence to adulthood: a population-based study. Rev Saúde Pública. 2007,41(1):69-75.

9. Mantilla-Toloza SC, Gómez-Conesa A, HidalgoMontesinos MD. Actividad física, tabaquismo y consumo de alcohol, en un grupo de estudiantes universitarios. Rev Salud Publica (Bogotá). 2011;13(5):748-58. 
10. Silva DAS, Petroski EL. Fatores associados ao nível de participação em atividades físicas em estudantes de uma universidade pública no Sul do Brasil. Cien Saude Colet. 2011;16(10):4087-94.

11. Marcondelli P, Costa THM, Schmitz BAS. Nível de atividade física e hábitos alimentares de universitários do $3^{\circ}$ ao $5^{\circ}$ semestres da área de saúde. Rev Nutr. 2008;21(1):39-47.

12. Cieslak F, Cavazza JF, Lazarotto L, Titski ACK, Stefanello JMF, Leite N. Análise da qualidade de vida e do nível de atividade física em universitários. Rev Educ Fis. 2012;23(2):251-60.

13. Ferreira NSA. As pesquisas denominadas "estado da arte". Educ Soc. 2002;23(79):257-72.

14. Romanowski JP, Ens RT. As pesquisas denominadas do tipo "Estado da Arte" em educação. Diálogo Educ. 2006;6(19):37-50.

15. Caspersen CJ, Powell KE, Christenson GM. Physical activity, exercise, and physical fitness: definitions and distinctions for health-related research. Public Health Rep. 1985;100(2):126-31.

16. Hallal PC, Knuth AG. Epidemiologia da atividade física e a aproximação necessária com as pesquisas qualitativas. Rev Bras Ciênc Esporte. 2011;33(1):181-92.

17. Rodrigues ESR, Cheik NC, Mayer AF. Nível de atividade física e tabagismo em universitários. Rev Saúde Pública. 2008;42(4):272-8.

18. Silva GSF, Bergamaschine R, Rosa M, Melo C, Miranda R, Filho MB. Avaliação do nível de atividade física de estudantes de graduação das áreas saúde/biológica. Rev Bra Med Esporte. 2007;13(1):39-42.

19. Quadros TMB, Petroski EL, Santos-Silva DA, Pinheiro-Gordia A. The prevalence of physical inactivity amongst Brazilian university students: its association with sociodemographic variables. Rev Salud Publica (Bogotá). 2009;11(5):724-33.

20. Guedes DP, Legnani RFS, Legnani E. Exercise motives in a sample of Brazilian university students. Motriz. 2013;19(3):590-6.

21. Batistella C. Abordagens contemporâneas do conceito de saúde. In: Fonseca AF, organizador. O território e o processo saúde-doença. Rio de Janeiro: Fiocruz; 2007. p. 54.

22. Castillo I, Molina-García J. Adiposidad corporal y bienestar psicológico: efectos de la actividad física en universitarios de Valencia, España. Rev Panam Salud Publica. 2009;26(4):334-40.

23. Dias C, Corte-Real N, Corredeira R, Barreiros A, Bastos T, Fonseca AM. A prática desportiva dos estudantes universitários e suas relaçóes com as autopercepçóes físicas, bem-estar subjectivo e felicidade. Estud Psicol (Natal). 2008;13(3):223-32.

24. Sousa TF. Atividades físicas praticadas no lazer por universitários de uma instituição pública do Nordeste do Brasil. Rev Bras Ativ Fís e Saúde. 2012;17(2):125-31.

25. Petroianu A, Santos BMR, Gonçalves RM. Deficiência visual e prática esportiva em estudantes de medicina e de educação física. RBM Rev Bras Med. 2004;61(11):726-29.
26. Nahas MV, Barros MVG, Francalacci V. O pentáculo do bem-estar: base conceitual para avaliação do estilo de vida de indivíduos ou grupos. Rev Bras Ativ Fís Saúde. 2000;5(2):48-59.

27. Colares V, Franca C, Gonzalez E. Condutas de saúde entre universitários: diferenças entre gêneros. Cad Saúde Pública. 2009;25(3):521-8

28. Brandão MP, Pimentel FL, Cardoso MF. Impact of academic exposure on health status of university students. Rev Saúde Pública. 2011;45(1):49-58.

29. Franca C, Colares V. Estudo comparativo de condutas de saúde entre universitários no início e no final do curso. Rev Saúde Pública. 2008;42(3):420-27.

30. García-Laguna DG, García-Salamanca GP, Tapiero-Paipa YT, Caballero DMR. Determinantes de los estilos de vida y su implicación en la salud de jóvenes universitarios. Hacia Promoc Salud. 2012;17(2):169-85.

31. Ferrari EP, Silva DAS, Petroski EL. Associação entre percepção da imagem corporal e estágios de mudança de comportamento em acadêmicos de educação física. Rev Bras Cineantropom Desempenho Hum. 2012;14(5):535-44.

32. Rech CR, Araújo ED., Vanat JDR. Autopercepção da imagem corporal em estudantes do curso de Educação Física. Rev Bras Educ Fís Esporte. 2010;24(2):285-92.

33. Ferrari EP, Gordia AP, Martins CR, Silva DA, Quadros TM, Petroski EL. Insatisfação com a imagem corporal e relação com o nível de atividade física e estado nutricional em universitários. Motricidade. 2012;8(3):52-8.

34. Simão $M$, Hayashida $M$, Santos $C B$, Cesarino EJ, Nogueira MS. Hipertensão arterial entre universitários da cidade de Lubango, Angola. Rev Latinoam Enferm. 2008;16(4):672-8.

35. Vilarinho RMF, Lisboa MTL, Thiré PK, França PV. Prevalência de fatores de risco de natureza modificável para a ocorrência de diabetes mellitus tipo 2. Esc Anna Nery Rev Enferm. 2008;12(3):452-56.

36. Belli T, Coelho FGM, Ferreira AS, Moura RF, Queiroga MR, Luciano E. Impacto do exercício agudo no perfil metabólico pós-prandial em adultos fisicamente aptos. Rev Bras Cineantropom Desempenho Hum. 2009;11(3):314-19.

37. Cordero RE, Pagavino D, Hernandéz CI, Contrera MI, García P, Sifontes ZM, et al. Biomarcadores séricos del estado de salud en jóvenes universitarios de acuerdo a su nivel de actividad física. Rev Fac Med (Caracas). 2008;31(1):29-36.

38. Ramalho AA, Dalamaria T, Souza OF. Consumo regular de frutas e hortaliças por estudantes universitários em Rio Branco, Acre, Brasil: prevalência e fatores associados. Cad Saúde Pública. 2012;28(7):1405-13.

39. Simão CB, Nahas MV, Oliveira ESA. Atividade física habitual, hábitos alimentares e prevalência de sobrepeso e obesidade em universitários da Universidade do Planalto Catarinense - Uniplac, Lages. SC. Rev Bras Ativ Fís Saúde. 2006;11(1)3-12.

40. Santos JS, Xavier AAO, Rieis EF, Costabeber I, Emanuelli T. Consumo de produtos de origem animal por universitários 
em Santa Maria, RS. Nutrire Rev Soc Bras Aliment Nutr. 2006;31(2):13-24.

41. Paixão LA, Dias RMR., Prado WL. Estilo de vida e estado nutricional de universitários ingressantes em cursos da área de saúde do Recife/PE. Rev Bras Ativ Fís Saúde. 2010;15(3):145-50.

42. Madureira AS, Corseuil HX, Pelegrini A, Petroski EL. Associaçáo entre estágios de mudança de comportamento relacionados à atividade física e estado nutricional em universitários. Cad Saúde Pública. 2009;25(10):2139-46.

43. Reis FB, Ciconelli RM, Faloppa F. Pesquisa Científica: a importância da metodologia. Rev Bras Ortop. 2002;37(3):51-5.

44. Turato ER. Métodos qualitativos e quantitativos na área de saúde: definiçóes, diferenças e seus objetos de pesquisa. Rev Saúde Pública. 2005;39(3):507-14.

45. Landim FLP, Laurinho LA, Lira RCM, Santos ZMSA. Uma reflexão sobre as abordagens em pesquisa com ênfase na integração qualitativo-quantitativa. Rev Bras Promoç Saúde. 2006;19(1):53-8.

46. González AM, Tamayo OE. Obstáculos cognitivo-emotivos para la realización de actividad física en estudiantes universitarios. Rev Latinoam Cienc Soc Niñez Juv. 2012;10(1):379-96.

47. Bourdieu P. Questóes de sociologia. Rio de Janeiro: Marco Zero; 1983.

48. Araújo FMB, Alves EM, Cruz MP. Algumas reflexóes em torno dos conceitos de campo e de habitus na obra de Pierre Bourdieu. Rev Perspect Cienc Tecnol. 2009;1(1):31-40.

49. Almeida Filho N. Intersetorialidade, transdisciplinaridade e saúde coletiva: atualizando um debate em aberto. Rev Adm Pública. 2000;34(6):11-34.

50. Pitanga FJG. Epidemiologia, atividade física e saúde. Rev Bras Ciênc Mov. 2002;10(3):49-54.

51. Fontes ACD, Vianna RPT. Prevalência e fatores associados ao baixo nível de atividade física entre estudantes universitários de uma universidade pública da região Nordeste, Brasil. Rev Bras Epidemiol. 2009;12(1):20-9.

52. Omram AR. The epidemiologic transition: a theory of the epidemiology of population change. Bull World Health Organ. 2001;79(2):509-38.

53. Coutinho RX, Soares MC, Folmer V, Puntel RL. Análise da produção de conhecimento da Educação Física brasileira sobre o cotidiano escolar. RBPG. 2012;9(17):491-516.

\section{Como citar este artigo:}

Cardoso GMP, Veras RM, Coelho MTAD, Figueredo WN. Vida universitária e atividade física: um estudo sobre a produção acadêmica. Rev. Aten. Saúde. 2017;15(52):78-88. 